\title{
Role of ubiquitin-conjugating enzyme E2T in the carcinogenesis and progression of pancreatic cancer
}

\author{
YUAN-WEN ZHENG ${ }^{1}$, PENG-FEI GAO ${ }^{2}$, MING-ZE MA $^{3}$, YONG CHEN $^{4}$ and CHUN-YOU LI ${ }^{1}$ \\ ${ }^{1}$ Department of Organ Transplantation and Second Hepatobiliary Surgery, Shandong Provincial Hospital \\ Affiliated to Shandong First Medical University; ${ }^{2}$ School of Medicine of Shandong University, Shandong University; \\ Departments of ${ }^{3}$ Infectious Diseases and ${ }^{4}$ Gastroenterology, Shandong Provincial Hospital \\ Affiliated to Shandong University, Jinan, Shandong 250021, P.R. China
}

Received January 29, 2019; Accepted October 22, 2019

DOI: $10.3892 / 01.2020 .11644$

\begin{abstract}
Ubiquitin-conjugating enzyme E2T (UBE2T) is a recently discovered oncogenic protein. Numerous studies reported that UBE2T is highly expressed in various types of human cancer; however, its role in the carcinogenesis and progression of pancreatic cancer remains unknown. The aim of the present study was to investigate the role of UBE2T in pancreatic cancer progression through in vitro experiments in pancreatic cancer tissues and cell lines. The results obtained in the present study demonstrated that UBE2T served an important role in the initiation and progression of pancreatic cancer. Furthermore, increased expression of UBE2T in human pancreatic cancer tissues and pancreatic cancer cells was observed compared with normal tissues and cells. The effect of upregulating and downregulating UBE2T in pancreatic cancer cell lines was investigated using the MTT, wound-healing and migration and invasion assays. The results demonstrated that overexpression of UBE2T significantly promoted pancreatic cancer cell proliferation, migration and invasion compared with controls. However, UBE2T downregulation resulted in the inhibition of pancreatic cancer cell proliferation, migration and invasion. In addition, the results demonstrated that UBE2T may promote the epithelial mesenchymal transition in pancreatic cancer cells.
\end{abstract}

\section{Introduction}

Pancreatic cancer is the fifth common malignant tumor in the gastrointestinal tract, with the incidence rate rising four times in the last twenty years in China (1).

Correspondence to: Professor Chun-You Li, Department of Organ Transplantation and Second Hepatobiliary Surgery, Shandong Provincial Hospital Affiliated to Shandong First Medical University, 324 Jingwu Road, Jinan, Shandong 250021, P.R. China

E-mail: chunyoudr@163.com

Key words: pancreatic cancer, epithelial mesenchymal transition, ubiquitin-conjugating enzyme E2T, invasion, proliferation
Furthermore, most patients with pancreatic cancer are diagnosed with ductal adenocarcinoma, which account for $90 \%$ of pancreatic cancer (1). Due to its high degree of malignancy, the prognosis of patients with pancreatic cancer is very poor. The global average annual incidence is 230,000, with an overall 5-year survival rate of $<5 \%(1,2)$. At present, radical resection remains the best therapeutic option for patients with early stage pancreatic cancer; however, due to the special anatomy of the pancreas, early symptoms of pancreatic cancer remain discrete in most patients and the early diagnosis is therefore difficult (2). The majority of patients are diagnosed at an advanced stage and cannot receive the optimal treatment strategy. The early diagnosis of pancreatic cancer is therefore essential (2-4).

UBE2T has been recently discovered as an oncogenic protein that belongs to the family of ubiquitin-conjugating enzymes (E2) (5). Ubiquitin can be transferred to E2 enzymes via the ubiquitin-activating enzyme (E1), and transferred to substrate proteins by ubiquitin ligase (E3) (5). The protein encoded by the UBE2T gene consists of 197 amino acids and catalyzes the covalent attachment of ubiquitin to protein substrates (6). Previous studies reported that detecting UBE2T expression could directly detect Fanconi anemia (FA) and interfere with the DNA damage-repair response $(5,6)$. Two transcript variants encoding different isoforms have been identified for this gene (6). There are two isoforms including NP-054895.1 and NP-001297255.1, which consist of 197 amino acids and 167 amino acids, respectively. Some of the UBE2T gene-associated pathways are the FA and the ubiquitin proteasome pathway (6). Data from Gene Ontology reported that UBE2T gene is associated with 'chromatin binding' and 'ubiquitin-protein transferase activity' (6) biological processes. Furthermore, the UBE2T gene participates in the protein monoubiquitination catalysis and is involved in the mitomycin-C induced DNA repair (5). In addition, UBE2T protein acts as a specific E2 enzyme for the FA core complex by binding to the E3 ligase named FA Complementation Group L (FANCL) and catalyzing the monoubiquitination of FA Complementation Group D2, which are crucial steps in DNA damage repair (6). The UBE2T gene is also involved in FANCL and FA Complementation Group I monoubiquitination (6). It has been reported that UBE2T might contribute 
to the ubiquitination and degradation of breast cancer type 1 susceptibility protein homolog, which serves an important role in regulating breast cancer cell proliferation and metastasis (7). Furthermore, the reactome pathways in which the UBE2T gene is involved include 'DNA repair', 'Fanconi anemia pathway', 'Metabolism of proteins', 'Post-translational protein modification', 'Protein ubiquitination' and 'synthesis of active ubiquitin-roles of E1 and E2 enzymes' (8). Whether UBE2T serves a role in pancreatic cancer remains unclear.

The present study investigated the role of UBE2T in pancreatic cancer initiation and progression. The results demonstrated that UBE2T is significantly upregulated in human pancreatic cancer tissues compared with normal tissues. Furthermore, the results demonstrated that high expression of UBE2T may promote proliferation, invasion and metastasis of pancreatic cancer cells. In addition, UBE2T may promote epithelial-mesenchymal transition (EMT). These data suggested that UBE2T may be considered as an oncogenic protein and a potential therapeutic target in pancreatic cancer.

\section{Materials and methods}

Tissues collection. A total of 121 patients, 76 males and 45 females, with pancreatic cancer were included in this study, aging from 36-78 years old (average age, 44.7 years). Pancreatic cancer resection tissues and normal adjacent tissues were collected from the same patients who underwent surgery at the Department of Organ Transplantation and Second Hepatobiliary Surgery of Shandong Provincial Hospital Affiliated to Shandong University between July 2009 and July 2017. The fresh tissues were stored at liquid nitrogen immediately. The present study was approved by the Ethics Committee of Shandong Provincial Hospital and all patients provided signed informed consent.

Immunohistochemistry (IHC). IHC staining of UBE2T was performed on pancreatic cancer and normal tissues. The tissue sections were fixed using $10 \%$ formalin and dehydrated with ethanol of gradient concentration $(75,85$ and $95 \%$ ethanol for $90 \mathrm{~min}$ each at room temperature; waterfree alcohol I and waterfree alcohol II for $30 \mathrm{~min}$ each; immersion wax temperature $58-65^{\circ} \mathrm{C}$ ). Then the tissues sections were embedded with paraffin at $60^{\circ} \mathrm{C}$. The tissues sections were then cut into $5 \mu \mathrm{m}$ thickness slides and rehydrated using xylene and ethanol of gradient concentration. Besides, antigen retrieval was performed using citrate buffer for $20 \mathrm{~min}$ at $90^{\circ} \mathrm{C}$. Endogenous peroxidase activity was inhibited with $3 \%$ hydrogen peroxide for $30 \mathrm{~min}$ at room temperature. Tissues were incubated with a primary antibody against UBE2T (1:200 dilution; cat. no. ab179802; Abcam) for $15 \mathrm{~h}$ at $4^{\circ} \mathrm{C}$. Tissue slides were then incubated with secondary antibody (goat anti-rabbit IgG H\&L horseradish peroxidase-conjugated preadsorbed; 1:500; cat. no. ab7090; Abcam) at room temperature for $1 \mathrm{~h}$. Signal was visualized using 3'-diaminobenzidine that reacts with HRP-labeled secondary antibody. The chromogenic region was observed and images were captured using a fluorescence microscope (Leica Instrument Co., Ltd.; magnification, x200).

Cell lines and cell culture. The human pancreatic cancer cell lines MIApaca-2 and Bxpc-3 were purchased from The Cell
Bank of Type Culture Collection of the Chinese Academy of Sciences. The cell line Panc-1 was donated by Joe Xinzhou, Shanghai. All cells were cultured with RPMI-1640 medium (MIApaca-2 and Bxpc-3 cells) or Dulbecco's Modified Eagle Medium (DMEM) (All purchased from Hyclone; GE Healthcare Life Sciences) for panc-1 cells, supplemented with $10 \%$ fetal bovine serum (FBS; Gibco; Thermo Fisher Scientific, Inc.) and 1x penicillin (100 IU/ml)/streptomycin $(100 \mu \mathrm{g} / \mathrm{ml})$ and placed at $37^{\circ} \mathrm{C}$ in a humidified incubator containing $5 \% \mathrm{CO}_{2}$.

Cells transfection. All the cells $\left(5 \times 10^{4} / \mathrm{ml}\right)$ were cultured in $6-\mathrm{cm}$ culture dishes without penicillin/streptomycin. Plasmid (2 $\mu \mathrm{g}$; purchased from GeneCopoeia, Inc.) transfection was performed by the lentivirus transfection system, using REV, GAG and VSV package vectors and Lipofectamine ${ }^{\mathrm{TM}} 2000$ (Invitrogen; Thermo Fisher Scientific, Inc.), according to the manufacturer's instructions, once cell density reached $\sim 80 \%$ for $\sim 48 \mathrm{~h}$. Cells were divided into four groups, according to the plasmid transfected: Psuper, psuper-UBE2TD, HA-psuper and HA-UBE2T-psuper. HA tag is a tag protein that can be fused to target gene and detected using HA tags. HA-UBE2T is the recombinant plasmid, which contains the HA tag and UBE2T ORF clone, in order to better observe the experimental results. The transfection efficiency is presented in Fig. S1. The following experiments were performed $48 \mathrm{~h}$ after transfection.

Cells were also transfected with shRNAs, the sequences were as follows: shUBE2T-A, 5'-GATCCCCAGACCAAA TGGATGACCTGTTCAAGAGACAGGTCATCCATTTGG TCTTTTTTGGAAA-3'; shUBE2T-B, 5'-GATCCCCTG CCAGACAGTGGACAGAGTTCAAGAGACTCTGTCCAC TGTCTGGCATTTTTGGAAA-3' (GENEWIZ, Inc.). The following experiments were performed $48 \mathrm{~h}$ after transfection.

Western blotting. Once cell density reached $\sim 80 \%$, cells were lysed using RIPA lysis buffer (Beyotime Institute of Biotechnology) supplemented with protease inhibitor cocktail on ice. Cell lysates were centrifuged at 12,000 x $\mathrm{g}$ for $30 \mathrm{~min}$ at $4^{\circ} \mathrm{C}$ following shaking and sonication every $1 \mathrm{~min}$ for three times on ice. Protein concentration detection was carried out using the BCA assay (Beijing Solarbio Science \& Technology Co., Ltd.) Supernatant was collected and mixed with 5X loading buffer (contain SDS and Bromphenol blue) and diluted to a final concentration of $1 \mathrm{X}$ sample buffer (from $5 \mathrm{X}$ buffer dilution). The samples were boiled at $95^{\circ} \mathrm{C}$ for $\sim 5 \mathrm{~min}$, in order to denature the lysate protein. Proteins $(40 \mu \mathrm{g})$ were separated by $10 \%$ SDS-PAGE gels and transferred onto polyvinylidene fluoride membranes. Membranes were blocked in $5 \%$ bovine serum albumin (BSA; $5 \mathrm{~g} / 1$, purchased from Sigma-Aldrich; Merck KGaA) for $1 \mathrm{~h}$ at room temperature, and incubated with primary antibody diluted in 5\% BSA and against $\beta$-actin $(1: 1,000$; cat. no. ab69512; Abcam), UBE2T (1:1,000; cat. no. ab179802; Abcam), vimentin (1:1,000; cat. no. 39325; Cell Signaling Technology, Inc.), E-cadherin (1:1,000; cat. no. 14472; Cell Signaling Technology, Inc.), N-cadherin (1:1,000; cat. no. 13116; Cell Signaling Technology, Inc.) and $\alpha$-catenin (1:50,000; cat. no. ab64479; Abcam) overnight at $4^{\circ} \mathrm{C}$. Membranes were washed three times with TBST $(0.2 \%$ Tween) and incubated with secondary antibodies: (goat anti-rabbit IgG H\&L DyLight488 (cat. no. ab96899; Abcam) and goat 
A

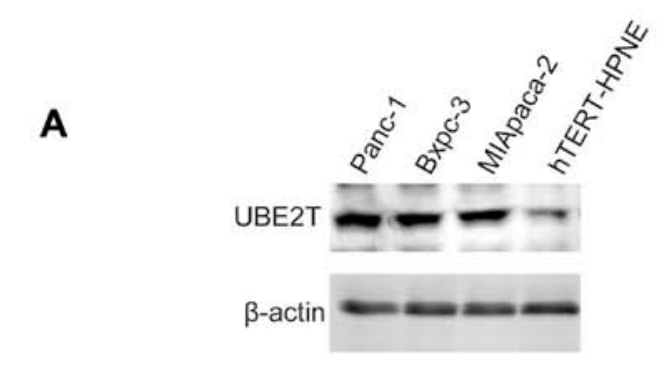

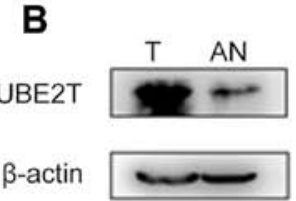

1

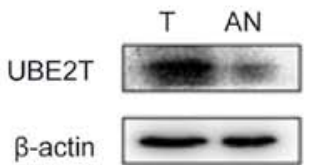

6

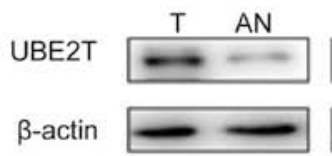

11

$\mathrm{T}=$ tumor

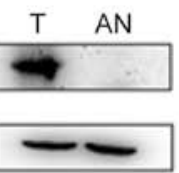

2

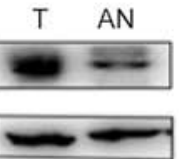

7

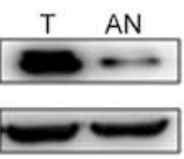

12

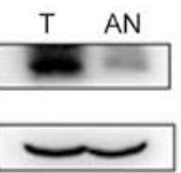

3

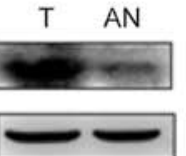

8

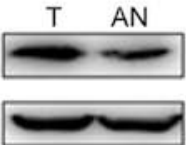

13

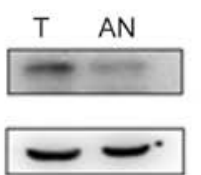

4

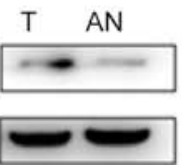

9

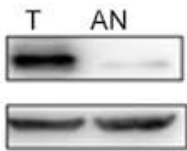

14

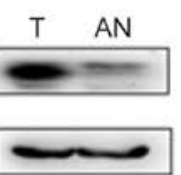

5

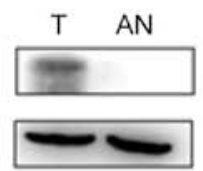

10

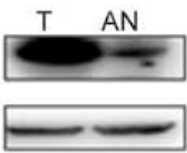

15

C

AN=Adjacent Non-tumor tissues

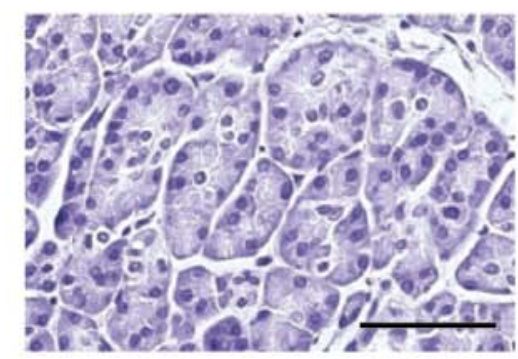

Normal (scale bar $=100 \mu \mathrm{m})$

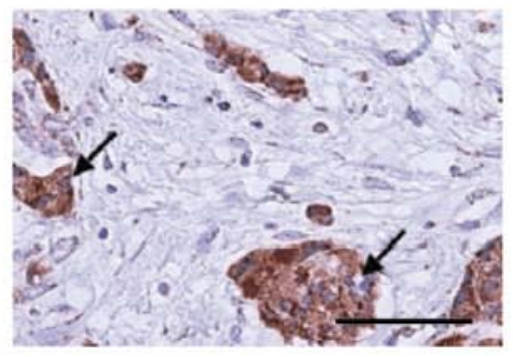

Tumor $\quad($ scale bar $=100 \mu \mathrm{m})$

Figure 1. UBE2T was upregulated in pancreatic cancer cells and tissues. (A) Western blotting demonstrated that UBE2T was upregulated in three pancreatic cancer cell lines. (B) UBE2T protein expression in pancreatic cancer tissues (15 cases) was increased compared with adjacent normal tissues. (C) Immunohistochemistry demonstrated that UBE2T expression was higher in pancreatic cancer tissues than in adjacent normal tissues. Arrows point to tumor cells. Magnification, x200. AN, adjacent normal tissue; UBE2T, ubiquitin-conjugating enzyme E2T; T, tumor tissue.

anti-mouse IgG H\&L DyLight594 (cat. no. ab96881; Abcam) (all 1:10,000) for $1 \mathrm{~h}$ at room temperature. Membranes were washed three times with TBST and bands were detected using Odessay Imaging system (supplied by LI-COR Biosciences).

MTT assay. In order to detect the effects of UBE2T on cell proliferation, transfected pancreatic cancer cells were seeded into 96-well plate at a density of 3,000 cells per well and placed at $37^{\circ} \mathrm{C}$ in a humidified incubator containing $5 \% \mathrm{CO}_{2}$ for $48 \mathrm{~h}$. Six biological replicates were analyzed per group and a blank well was also set up with only medium. Cells were cultured for five days until a confluence of $80-90 \%$ was reached. Cells were incubated with MTT reagent $(10 \mu 1 /$ well) and placed for $4 \mathrm{~h}$ at $37^{\circ} \mathrm{C}$ in the dark. Supernatant was removed and purple formazan was dissolved with $150 \mu \mathrm{l}$ DMSO. The plate was oscillated for $10 \mathrm{~min}$ and the optical density was measured at $570 \mathrm{~nm}$ with a microplate reader.

Wound-healing assay. All transfected pancreatic cancer cell lines were cultured in $6-\mathrm{cm}$ culture dishes at a density of $5 \times 10^{4} / \mathrm{ml}$. Cells were scratched using a 200- $\mu 1$ tip, floating cells were washed with Hank's Balanced Salt Solution (Thermo Fisher Scientific, Inc.) and new medium (DMEM contain $10 \%$ bovine serum) was added. Cells were incubated for $48 \mathrm{~h}$ at $37^{\circ} \mathrm{C}$. The wound width was observed and recorded every $24 \mathrm{~h}$ under a microscope (magnification, x100). The results were observed using the Zeiss inverted fluorescence microscope (Zeiss AG). 
A

MIApaca-2

Bxpc-3

Panc-1

UBE2T
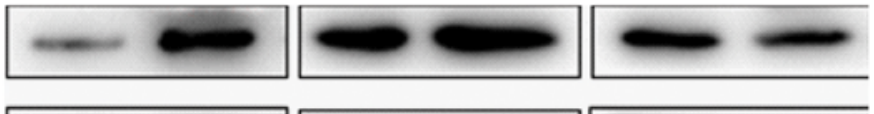

$\beta$-actin
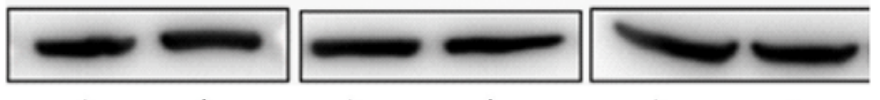

$0^{0}$

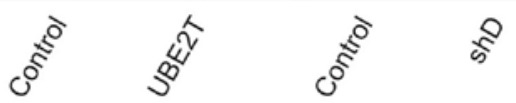

B

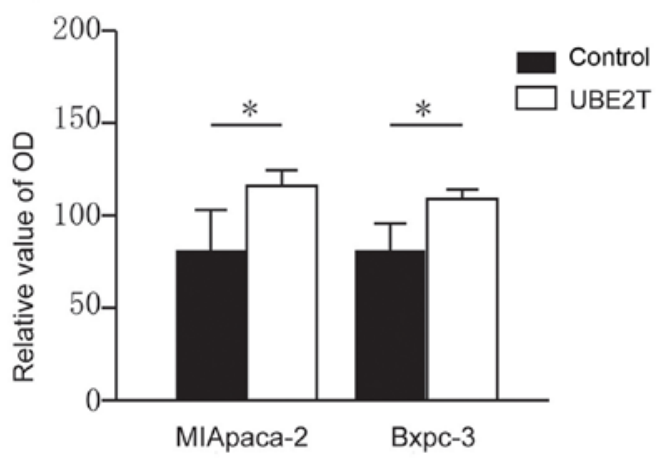

D

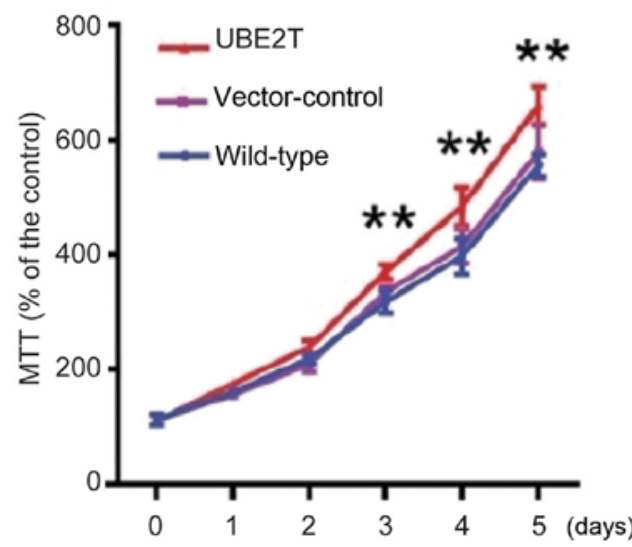

C

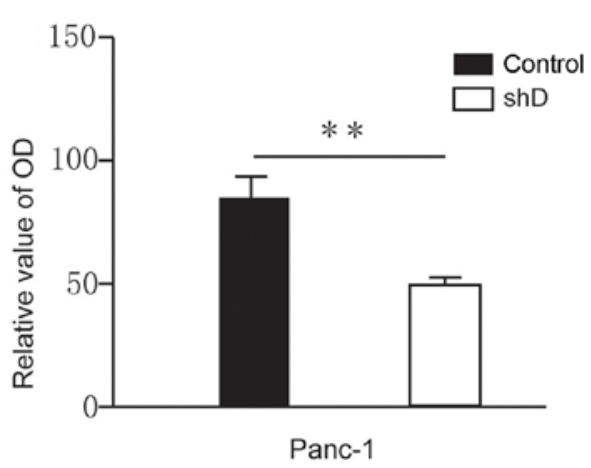

Bxpc-3

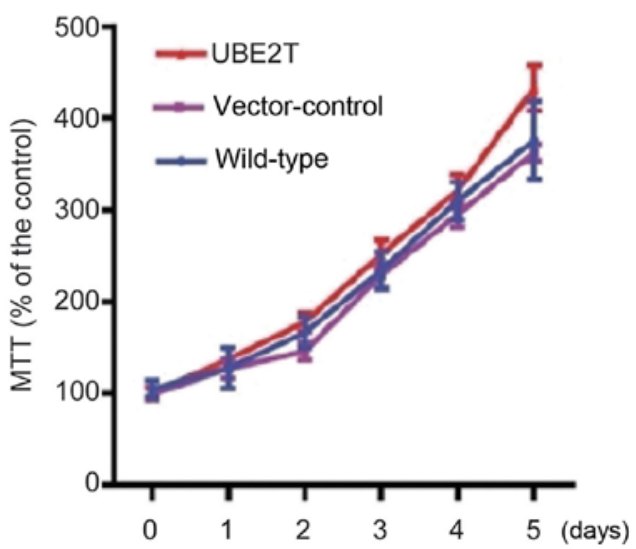

Figure 2. UBE2T promoted pancreatic cancer cell proliferation. (A) Western blotting demonstrated that UBE2T protein expression was higher in cells lines overexpressing UBE2T compared with the control group. (B) The MTT assay demonstrated that the proliferation of MIApaca-2 and Bxpc-3 cells overexpressing UBE2T was higher compared with the control cells. ${ }^{*} \mathrm{P}<0.05$. (C) Panc-1 cell proliferation was decreased following transfection with shRNA compared with the control cells. ${ }^{* * *} \mathrm{P}<0.01$. (D) The MTT assay demonstrated that MIApaca-2 and Bxpc-3 cell proliferation was higher following UBE2T overexpression compared with control cells for up to five days. shD, pSuper-shUBE2T.D (shRNA silence UBE2T pSuper); OD, optical density; UBE2T, ubiquitin-conjugating enzyme E2T.

Cell invasion and migration assay. Transwell (pore size, $8 \mu \mathrm{m}$ ) chambers (BD Biosciences) were coated with Matrigel and incubated for $30 \mathrm{~min}$ at $37^{\circ} \mathrm{C}$ and were inserted into a 24 -well plate. Transfected pancreatic cancer cells were cultured with RPMI1640 medium or DMEM supplemented with $1 \%$ FBS. Cell suspension $\left(\sim 8 \times 10^{4}\right.$ cells $\left./ 200 \mu \mathrm{l}\right)$ was added in the upper chamber and $500 \mu \mathrm{l}$ corresponding medium supplemented with $10 \%$ FBS was added into the bottom chamber. Cells were cultured in the incubator for $48 \mathrm{~h}$ at $37^{\circ} \mathrm{C}$ The cells on the upper side of the membrane were removed and the cells that invaded through the membrane were fixed with methanol for $30 \mathrm{~min}$ at room temperature and stained with $1 \%$ crystal violet for $30 \mathrm{~min}$ at room temperature Five random fields from each membrane were counted under a Zeiss inverted fluorescence microscope at $\times 200$ magnification.
The protocol for migration assay was identical to the invasion assay; however, Transwell chambers were not coated with Matrigel.

Statistical analysis. All data are expressed as the means \pm standard deviation. Differences between the two groups were analyzed using a Student t-test. $\mathrm{P}<0.05$ was considered to indicate a statistically significant difference.

\section{Results}

UBE2T is upregulated in pancreatic cancer cells and tissues. The expression of UBE2T was detected in the three pancreatic cancer cell lines (MIApaca-2, Bxpc-3 and Panc-1) and the normal pancreatic cell line (hTERT-HPNE cells). The 
A

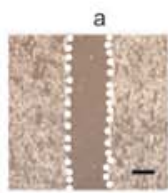

Oh

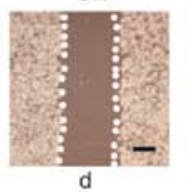

B

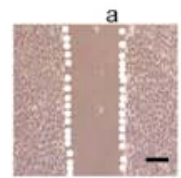

Oh

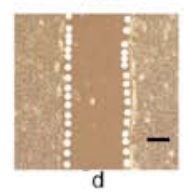

C
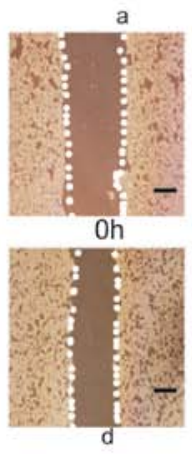

MIApaca-2

b

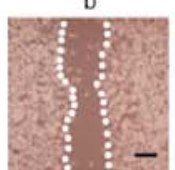

$24 \mathrm{~h}$

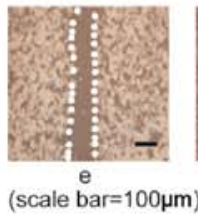

Bxpc-3

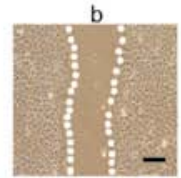

$24 h$

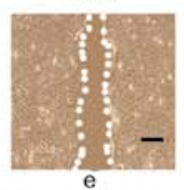

(scale bar $=100 \mu \mathrm{m}$ )

Panc-1
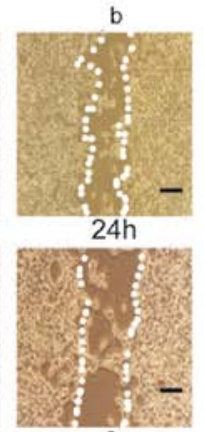

(scale bar $\stackrel{e}{e}=100 \mu \mathrm{m}$ )

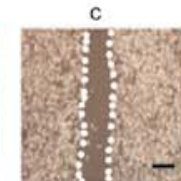

$48 \mathrm{~h}$
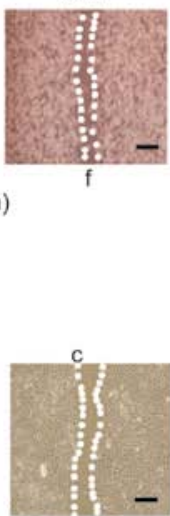

$48 \mathrm{~h}$
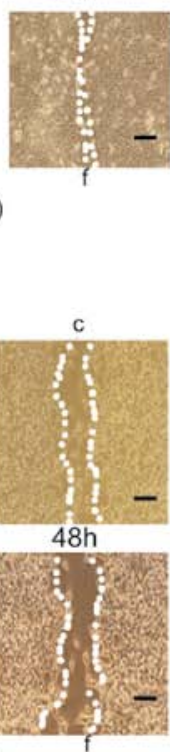

D

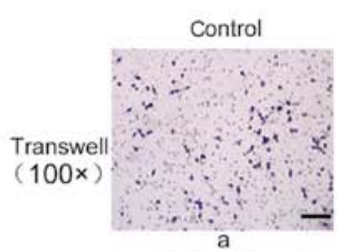

MIApaca-2

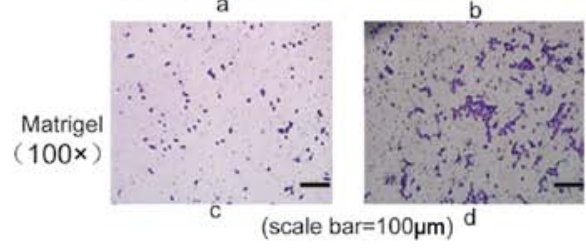

E
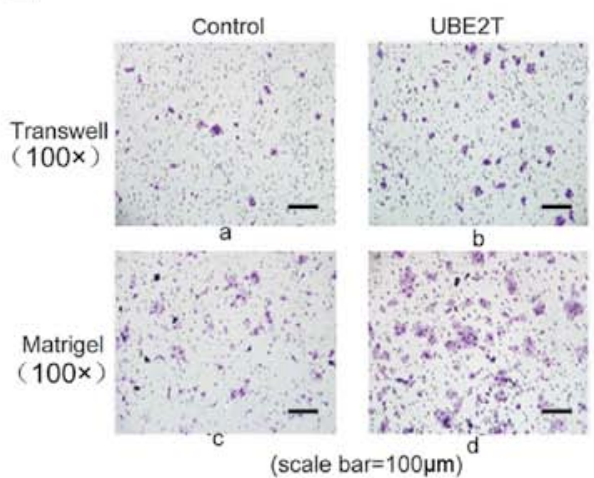

$\mathbf{F}$
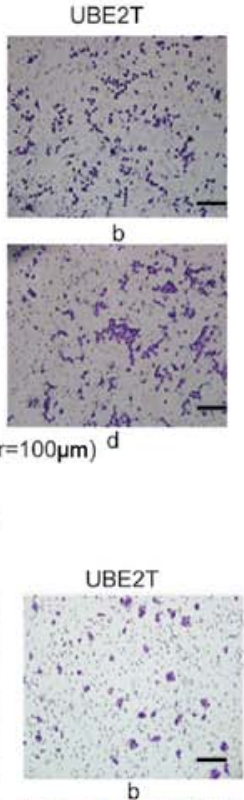

\section{(1)}




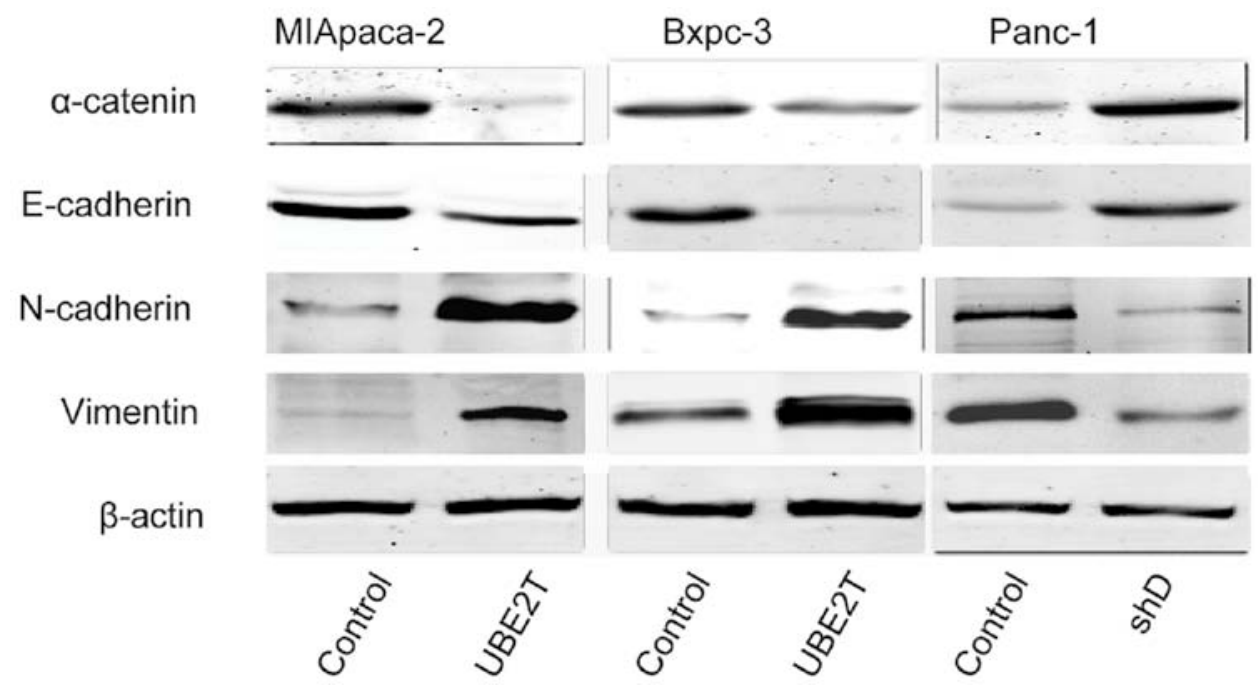

Figure 4. UBE2T overexpression promoted the epithelial-mesenchymal transition in pancreatic cancer cells. Results demonstrated that the protein expression of the epithelial markers E-cadherin and $\alpha$-catenin was decreased, and that the protein expression of the mesenchymal markers vimentin and $N$-cadherin was increased in cells overexpressing UBE2T. In Panc1 transfected with shRNA-UBE2T, the protein expression of vimentin and N-cadherin was decreased and the protein expression of E-cadherin and $\alpha$-catenin was increased. UBE2T, ubiquitin-conjugating enzyme E2T; shD, pSuper-shUBE2T.D (shRNA silence UBE2T pSuper).

shelf for $25 \mathrm{~min}$. The mixture in the tube was added to the petri dish, which was replaced with serum-containing medium after $6 \mathrm{~h}$. A 10-ml disposable syringe was used to aspirate the old culture medium, which was filtered with a disposable filter, and dispensed into a 15-ml disposable centrifuge tube.

The MTT assay was then used to detect the effect of UBE2T on pancreatic cancer cell proliferation. The results demonstrated that the proliferation of MIApaca-2 and Bxpc-3 cell lines overexpressing UBE2T was increased compared with normal cells (Fig. 2B and D). Conversely, the proliferation of Panc-1 cells transfected with shRNA-UBE2T was decreased compared with control cells.

UBE2T promotes pancreatic cancer cell invasion and migration. The results demonstrated that UBE2T overexpression could promote pancreatic cell proliferation. Subsequently, wound healing and Transwell assays were performed to measure the effect of UBE2T on pancreatic cancer cell invasion and migration.

The results from the wound healing assay demonstrated that UBE2T overexpression improved the migratory ability of MIApaca2 (Fig. 3A-d-f) and Bxpc3 (Fig. 3 B-d-f) cells compared with corresponding control cells (non-transfected cells) (Fig. 3A-a-c and B-a-c). However, the migratory ability of Panc-1 cells transfected with shRNA-UBE2T (Fig. 3C-d-f) was inhibited compared with control group (Fig. 3Ca-c). In order to further confirm these results, Transwell assays were performed. The results demonstrated that UBE2T overexpression (Fig. 3D-d; Fig. 3E-d) promoted pancreatic cancer cell invasion compared with control groups (Fig. 3D-c; Fig. 3E-c). The results demonstrated that UBE2T overexpression (Fig. 3D-b; Fig. 3E-b) promoted pancreatic cancer cell migration compared with control groups (Fig. 3D-a; Fig. 3E-a). However, the invasive ability of Panc-1 transfected with shRNA-UBE2T (Fig. 3F-d) was inhibited compared with the control group (Fig. 3F-c). Besides, the migration ability of Panc-1 transfected with shRNA-UBE2T (Fig. 3F-b) was inhibited compared with the control group (Fig. 3F-a). These findings indicated that UBE2T may promote pancreatic cancer cell invasion and migration.

UBE2T promotes EMT in pancreatic cancer cells. As EMT serves a crucial role in tumor development (9), the present study investigated the effect of UBE2T on the expression of epithelial and mesenchymal marker proteins via western blotting. The results demonstrated that the expression of the epithelial markers E-cadherin and $\alpha$-catenin was decreased whereas the expression of the mesenchymal markers vimentin and $\mathrm{N}$-cadherin was increased in pancreatic cells overexpressing UBE2T compared with the control group. Furthermore, in Panc-1 cells transfected with shRNA-UBE2T, the expression of mesenchymal markers was decreased whereas the expression of epithelial markers was increased (Fig. 4).

\section{Discussion}

Pancreatic cancer represents a type of solid tumor that is highly malignant and invasive (7). Furthermore, little is know about the pathogenesis of pancreatic cancer. It is therefore crucial to determine some genes that would be associated with the invasive and metastatic abilities of pancreatic cancer cells and to further investigate the mechanisms involved in the occurrence and development of this cancer.

Previous studies reported that UBE2T is highly expressed in bladder cancer, prostate cancer, gastric cancer and nasopharyngeal carcinoma (7-9), and that UBE2T is elevated in the majority of tumors of the digestive system. The present study demonstrated that UBE2T was upregulated in pancreatic cancer tissues and that UBE2T overexpression promoted cancer cell proliferation and invasion in vitro. The underlying mechanism of UBE2T on the promotion of malignant biological behavior in pancreatic cancer requires further investigation. 
EMT and mesenchymal-epithelial transformation are considered as key steps in cancer cell metastasis initiation or endpoint (10). Previous studies on prostate cancer reported that UBE2T overexpression induces the upregulation of mesenchymal markers, including vimentin, fibronectin and $\alpha$-smooth muscle actin, and the downregulation of epithelial markers, including E-cadherin (9). It has been reported that UBE2T can inhibit the degradation of vimentin caused by F-box and WD repeat domain containing 7 (FBXW7), which is a tumor suppressor (7). The molecular mechanisms underlying the inhibition of vimentin degradation by UBE2T may be due to the influence from the interconnection between FBXW7 and vimentin; and that the 95 amino acid site in UBE2T protein is the binding site between UBE2T and vimentin (8). However, to the best of our knowledge, the role of UBE2T in EMT regulation in pancreatic cancer has not yet been reported. The results from the present study reported that UBE2T overexpression could promote EMT in pancreatic cancer cells, which was similar to findings from previous studies on prostate cancer $(8,10)$.

In conclusion, results from this study suggested that UBE2T may be considered as a potential therapeutic target in pancreatic cancer progression. In addition, UBE2T was demonstrated to promote pancreatic cancer cell invasion, which may be mediated through EMT regulation and other mechanisms. Although UBE2T may be considered as a potential diagnostic biomarker and therapeutic target in pancreatic cancer, further investigation is required to determine the underlying mechanisms involved in the regulation of tumor biological behavior by UBE2T.

\section{Acknowledgements}

The authors would like to thank Mrs Ling Li (Jinan Infectious Disease Hospital, Jinan, Shandong) for the help with the IHC staining assay.

\section{Funding}

The present study was supported by the Natural Foundation of Shandong Province (grant no. ZR2018BH003).

\section{Availability of data and materials}

The datasets used and/or analyzed during the current study are available from the corresponding author on reasonable request.

\section{Authors' contributions}

$\mathrm{YZ}$ and $\mathrm{CL}$ conceived the project and designed the experiments. PG and MM performed experiments and analyzed data. YC contributed to the conception and design of the work, as well as to the analysis and interpretation of data. YZ and CL wrote the manuscript. All authors accepted the revised version of the manuscript.

\section{Ethics approval and consent to participate}

The present study was approved by the Ethics Committee of Shandong Provincial Hospital and all patients provided signed informed consent.

\section{Patient consent for publication}

Not applicable.

\section{Competing interests}

The authors declare that they have no competing interests.

\section{References}

1. Jemal A, Siegel R, Ward E, Hao Y, Xu J and Thun MJ: Cancer statistics, 2009. CA Cancer J Clin 59: 225-249, 2009.

2. Hidalgo M: Pancreatic cancer. N Engl J Med 362: 1605-1617, 2010.

3. Witkowski ER, Smith JK and Tseng JF: Outcomes following resection of pancreatic cancer. J Surg Oncol 107: 97-103, 2013.

4. Hassan MM, Bondy ML, Wolff RA, Abbruzzese JL, Vauthey JN, Pisters PW, Evans DB, Khan R, Chou TH, Lenzi R, et al: Risk factors for pancreatic cancer: Case-control study. Am J Gastroenterol 102: 2696-2707, 2007.

5. Gong YQ, Peng D, Ning XH, Yang XY, Li XS, Zhou LQ and Guo YL: UBE2T silencing suppresses proliferation and induces cell cycle arrest and apoptosis in bladder cancer cells. Oncol Lett 12: 4485-4492, 2016.

6. Wen M, Kwon Y, Wang Y, Mao JH and Wei G: Elevated expression of UBE2T exhibits oncogenic properties in human prostate cancer. Oncotarget 6: 25226-25239, 2015.

7. Yu H, Xiang P, Pan Q, Huang Y, Xie N and Zhu W: Ubiquitin-Conjugating enzyme e2t is an independent prognostic factor and promotes gastric cancer progression. Tumour Biol 37: 11723-11732, 2016.

8. Hu W, Xiao L, Cao C, Hua S and Wu D: UBE2T promotes nasopharyngeal carcinoma cell proliferation, invasion, and metastasis by activating the AKT/GSK3 $\beta / \beta$-catenin pathway. Oncotarget 7: 15161-15172, 2016

9. Rickman KA, Lach FP, Abhyankar A, Donovan FX, Sanborn EM, Kennedy JA, Sougnez C, Gabriel SB, Elemento O, Chandrasekharappa SC, et al: Deficiency of UBE2T, the E2 ubiquitin ligase necessary for FANCD2 and FANCI ubiquitination, causes FA-T subtype of Fanconi anemia. Cell Rep 12: 35-41, 2015.

10. Brand TM, Iida M, Luthar N, Starr MM, Huppert EJ and Wheeler DL: Nuclear EGFR as a molecular target in cancer. Radiother Oncol 108: 370-377, 2013. 